\title{
Boundary control of hyperbolic conservation laws using a frequency domain approach
}

\author{
Xavier Litrico and Vincent Fromion
}

\begin{abstract}
The paper uses a frequency domain method for boundary control of hyperbolic conservation laws. We show that the transfer function of the hyperbolic system belongs to the Callier-Desoer algebra, for which the Nyquist theorem provides necessary and sufficient conditions for input-output closed-loop stability. We examine the link between input-output stability and exponential stability of the state. Specific results are then derived for the case of proportional boundary controllers. The results are illustrated in the case of boundary control of openchannel flow.
\end{abstract}

\section{INTRODUCTION}

Hyperbolic conservations laws are derived from physics of distributed parameter systems. We deal in this paper with systems represented by hyperbolic conservations laws with an independent time variable $t \in[0,+\infty)$ and an independent space variable on a finite interval $x \in[0, L]$, for which we derive stabilizing boundary controllers using a frequency domain approach.

This work is motivated by the problem of controlling an open-channel represented by Saint-Venant equations. These hyperbolic partial differential equations describe the dynamics of open-channel hydraulic systems, e.g. rivers, irrigation or drainage canals, sewers, etc., assuming one dimensional flow.

Many authors contributed on the control of open-channel hydraulic systems represented by Saint-Venant equations. Most of these works used a finite dimensional approximation of the system to design controllers. Recent approaches took into account the distributed feature of the system by a Riemann invariants approach [7]. This method provides a sufficient stability result for rectangular horizontal frictionless channels around a uniform flow regime. For more realistic cases, only vanishing perturbations can be considered [11]. This main limitation of the Riemann invariants method leads to consider an alternative method based on a frequency domain approach, which we have already developed in previous papers [10], [9], by considering only input-output stability. Here, we also study the Lyapunov-like behavior of the system for non zero initial conditions.

The objective of this paper is to link the Lyapunov approach with the frequency domain approach. The main results of the paper are as follows:

1) We provide a detailed characterization of the transfer matrix of the considered hyperbolic system, and show that it belongs to the class $\hat{\mathcal{B}}(\sigma)$ of Callier-Desoer [4],

$\mathrm{X}$. Litrico is with UMR G-EAU, Cemagref, Montpellier, France. xavier.litrico@cemagref.fr.

V. Fromion is with INRA-MIG, Jouy-en-Josas, France. vincent.fromionejouy.inra.fr.
2) We use Nyquist theorem to derive necessary and sufficient condition for input-output stability of boundary controlled hyperbolic systems,

3) We clarify the link between input-output and internal stability.

We also examine in detail the specific case of proportional diagonal boundary control and extend the results presented by [7]. These results are illustrated for boundary control of linearized Saint-Venant equations, representing open-channel flow around a given stationary regime.

\section{CONTROL PROBLEM STATEMENT}

\section{A. Control problem}

We consider the following linear system of hyperbolic conservation laws:

$$
\frac{\partial \xi}{\partial t}+\left(\begin{array}{cc}
0 & 1 \\
\alpha \beta & \alpha-\beta
\end{array}\right) \frac{\partial \xi}{\partial x}+\left(\begin{array}{cc}
0 & 0 \\
-\gamma & \delta
\end{array}\right) \xi=0
$$

where $t$ and $x$ are the two independent variables : a time variable $t \in[0,+\infty)$ and a space variable $x \in[0, L]$ on a finite interval, $\xi(t, x)=(h(t, x), q(t, x))^{T}:[0,+\infty) \times$ $[0, L] \rightarrow \Omega \in \mathbb{R}^{2}$ is the state of the system. $\alpha>\beta>0$, $\gamma \geq 0$ and $\delta \geq 0$ are positive real constants.

The two equations of system (1) can be interpreted as a mass conservation law with $h$ the conserved quantity and $q$ the flux. The second equation can then be interpreted as a momentum conservation law.

We consider the solutions of the Cauchy problem for the system (1) over $[0,+\infty) \times[0, L]$ under an initial condition $\xi(0, x)=\xi_{0}(x), x \in[0, L]$ and two boundary conditions of the form $q(t, 0)=q_{0}(t)$ and $q(t, L)=q_{L}(t), t \in[0,+\infty)$.

\section{B. Existence and well-posedness}

Following a classical approach, we introduce the bounded group $\mathbb{T}(t)$ on $\mathcal{L}_{2}\left([0, L], \mathbb{R}^{2}\right)$, generated by the following linear operator:

$$
A_{1} \xi=\left(\begin{array}{cc}
0 & 1 \\
\alpha \beta & \alpha-\beta
\end{array}\right) \frac{\partial \xi}{\partial x}+\left(\begin{array}{cc}
0 & 0 \\
-\gamma & \delta
\end{array}\right) \xi
$$

where $A_{1}$ is then defined on the domain in $\mathcal{L}_{2}\left([0, L], \mathbb{R}^{2}\right)$ consisting of functions $\xi \in H^{1}\left([0, L], \mathbb{R}^{2}\right)$ which satisfy the boundary conditions $C \xi(t, 0)=q_{0}(t)$ and $C \xi(t, L)=q_{L}(t)$ with $C=\left(\begin{array}{ll}0 & 1\end{array}\right) \cdot H^{1}\left([0, L], \mathbb{R}^{2}\right)$ corresponds to the Sobolev space of $\mathbb{R}^{2}$ functions whose derivatives (in generalized sense) are square integrable on $[0, L]$. 
1) Continuous solutions: Let us first recall that if $\xi_{0}(x)$ and $u(t)=\left(q_{0}(t), q_{L}(t)\right)^{T}$ are two continuously differentiable functions of their argument, it is then possible by Theorem 2.1 in [13] to claim that the solutions of system (1) are continuously differentiable with respect to its arguments, i.e., $\xi(t, x) \in \mathcal{C}^{1}\left([0, \infty),[0, L], \mathbb{R}^{2}\right)$. Furthermore there exist two finite constants $M>0$ and $\gamma$ such that for any $t \in$ $[0, \infty)$, any $\xi \in \mathcal{C}^{1}\left([0, L], \mathbb{R}^{2}\right)$ and any $u \in \mathcal{L}_{2}\left([0, t], \mathbb{R}^{2}\right) \cap$ $\mathcal{C}^{1}\left([0, t], \mathbb{R}^{2}\right)$, there exists a finite constant $K_{t}$ such that

$$
\|\xi(t, \cdot)\|_{\mathcal{L}_{2}\left([0, L], \mathbb{R}^{2}\right)} \leq M e^{\gamma t}\left\|\xi_{0}\right\|_{\mathcal{L}_{2}\left([0, L], \mathbb{R}^{2}\right)}+K_{t}\|u\|_{2} .
$$

2) Generalized solutions: Following this preliminary result and the fact that the continuous differentiable functions defined on any finite support are dense in $\mathcal{L}_{2}$, it is then possible to handle the inputs and the initial conditions in $\mathcal{L}_{2}\left([0, t], \mathbb{R}^{2}\right)$ and $\mathcal{L}_{2}\left([0, L], \mathbb{R}^{2}\right)$ respectively. We thus conclude that system (1) has a generalized solution in $\mathcal{C}\left([0, \infty), \mathcal{L}_{2}\left([0, L], \mathbb{R}^{2}\right)\right)$ for any $t \in[0, \infty)$, any $\xi_{0} \in$ $\mathcal{L}_{2}\left([0, L], \mathbb{R}^{2}\right)$ and any $\left(q_{0}, q_{L}\right) \in \mathcal{L}_{2}\left([0, t], \mathbb{R}^{2}\right)$. Let us recall that $\xi \in \mathcal{C}\left([0, \infty), \mathcal{L}_{2}\left([0, L], \mathbb{R}^{2}\right)\right)$ means that for every $t \geq 0 \xi(t, \cdot)$ belongs to $\mathcal{L}_{2}\left([0, L], \mathbb{R}^{2}\right)$ and $\lim _{h \rightarrow 0} \| \xi(t+$ $h, \cdot)-\xi(t, \cdot) \|_{\mathcal{L}_{2}\left([0, L], \mathbb{R}^{2}\right)}=0$. Furthermore, the solution of system (1) can be rewritten as

$$
\xi(t, \cdot)=\Phi(t) u^{(t)}+\mathbb{T}(t) \xi_{0}
$$

where $u^{(t)}$ denotes the restriction of $u$ to $[0, t]$ and where $\Phi(t)$ is a bounded linear operator defined from $\mathcal{L}_{2}\left([0, t], \mathbb{R}^{2}\right)$ into $\mathcal{L}_{2}\left([0, L], \mathbb{R}^{2}\right)$. Finally, the generalized solution also satisfies inequality (3) (see [14] for details).

It remains to ensure that the output of the system is welldefined, i.e., for any $t \in[0, \infty)$, any $\xi_{0} \in \mathcal{L}_{2}\left([0, L], \mathbb{R}^{2}\right)$ and any $\left(q_{0}, q_{L}\right) \in \mathcal{L}_{2}\left([0, t], \mathbb{R}^{2}\right), y(t)=(h(t, 0), h(t, L))$ belongs to $\mathcal{L}_{2}\left([0, t], \mathbb{R}^{2}\right)$. As in the case of the existence of generalized solutions, the main idea in this context is to use a density type argument. We do not develop the details of the proof since this proof can be easily adapted from the one associated to example 4.3.12 in [6].

\section{FREQUENCY DOMAIN ANALYSIS}

Using the results of section II, we know that the solutions of (1) are Laplace transformable, which enable us to use a frequency domain approach. We will show in the sequel that the transfer matrix of system (1) belongs to the CallierDesoer algebra [4], [5]. That ensures that the closed-loop system is well-defined and the validity of the Nyquist criteria, which provides necessary and sufficient conditions for stability of feedback system. We begin with some definitions.

\section{A. Definitions}

Let $\sigma \in \mathbb{R}$ be a given real number.

Definition 1 (Sets $\mathcal{A}(\sigma), \hat{\mathcal{A}}(\sigma), \mathcal{A}_{-}(\sigma)$ and $\hat{\mathcal{A}}_{-}(\sigma)$.): Let $\mathcal{A}(\sigma)$ denote the set of distributions $f$ such that:

$$
f(t)= \begin{cases}0 & \text { if } t<0 \\ \sum_{i=0}^{\infty} f_{i} \delta\left(t-t_{i}\right)+f_{a}(t) & \text { if } t \geq 0,\end{cases}
$$

where $f_{a}(t) e^{-\sigma t} \in L_{1}(0, \infty), \delta($.$) represents the unit delta$ distribution, $0 \leq t_{0}<t_{1}<\ldots$ and $f_{i}$ are real constants, and $\sum_{i=0}^{\infty}\left|f_{i}\right| e^{-\sigma t_{i}}<\infty$.
$\hat{\mathcal{A}}(\sigma)$ denotes the set of all functions $\hat{f}: \mathbb{C}^{+} \rightarrow \mathbb{C}$ that are Laplace transforms of elements of $\mathcal{A}(\sigma)$; they are analytic and bounded in $\Re(s) \geq \sigma$, where $\Re(s)$ denotes the real part of $s$. The sets $\mathcal{A}_{-}(\sigma)$ and $\hat{\mathcal{A}}_{-}(\sigma)$ are defined as:

$$
\mathcal{A}_{-}(\sigma)=\bigcup_{\sigma_{1}<\sigma} \mathcal{A}\left(\sigma_{1}\right) \text { and } \hat{\mathcal{A}}_{-}(\sigma)=\bigcup_{\sigma_{1}<\sigma} \hat{\mathcal{A}}\left(\sigma_{1}\right)
$$

$\hat{\mathcal{A}}_{-}^{\infty}(\sigma)$ denotes the set of elements $\hat{b} \in \hat{\mathcal{A}}_{-}(\sigma)$ being bounded away from zero at infinity in $\Re(s) \geq \sigma$.

Definition 2 (The Callier-Desoer class $\hat{\mathcal{B}}(\sigma)$ ): The set $\hat{\mathcal{B}}(\sigma)$ consists of all functions $\hat{f}=\hat{a} / \hat{b}$, where $\hat{a} \in \hat{\mathcal{A}}_{-}(\sigma)$ and $\hat{b} \in \hat{\mathcal{A}}_{-}^{\infty}(\sigma)$.

\section{B. Open-loop transfer matrix}

1) Input-output transfer matrix: The system's open-loop transfer matrix can be obtained by applying Laplace transform to the linear partial differential equations (1), and solving the resulting system of Ordinary Differential Equations in the variable $x$, parameterized by the Laplace variable $s$ [8]. In this case, using the classical relation $\frac{\hat{d f}}{d t}=s \hat{f}(s)-f(0)$ and after elementary manipulations, we get:

$$
\frac{\partial \hat{\xi}(s, x)}{\partial x}=A(s) \hat{\xi}(s, x)+B \xi(0, x)
$$

with

$$
\begin{aligned}
A(s) & =\frac{1}{\alpha \beta}\left(\begin{array}{cc}
\alpha-\beta) s+\gamma & -s-\delta \\
-\alpha \beta s & 0
\end{array}\right) \\
B & =\frac{1}{\alpha \beta}\left(\begin{array}{cc}
(\beta-\alpha) & 1 \\
\alpha \beta & 0
\end{array}\right) .
\end{aligned}
$$

The general solution of (4) is then given by:

$$
\hat{\xi}(s, x)=e^{A(s) x}\left[\hat{\xi}(s, 0)+\bar{\xi}_{0}(s, x)\right]
$$

with $\bar{\xi}_{0}(s, x)=\int_{0}^{x} e^{-A(s) v} B \xi(0, v) d v$.

The state $\hat{\xi}(s, x)$ is then obtained with the transition matrix $\Gamma(s, x)=e^{A(s) x}$ acting on the sum of two terms: the first one $\hat{\xi}(s, 0)$ is the boundary condition in $x=0$, and the second one $\bar{\xi}_{0}(s, x)$ is linked to the initial condition at $t=0$.

Then, using an algebraic manipulation to specify the boundary inputs $\hat{u}(s)=(\hat{q}(s, 0), \hat{q}(s, L))^{T}$ and outputs $\hat{y}(s)=(\hat{h}(s, 0), \hat{h}(s, L))^{T}$, we get the following representation:

$$
\hat{y}(s)=P(s) \hat{u}(s)+P_{0}(s) \bar{\xi}_{0}(s, L)
$$

where $P_{0}(s)=P(s)\left(\begin{array}{ll}0 & 1 \\ 0 & 0\end{array}\right)-\left(\begin{array}{ll}1 & 0 \\ 0 & 0\end{array}\right)$, and

$$
P(s)=\left(\begin{array}{cc}
\frac{\lambda_{2} e^{\lambda_{1} L}-\lambda_{1} e^{\lambda_{2} L}}{s\left(e^{\lambda_{2} L}-e^{\lambda_{1} L}\right)} & \frac{\lambda_{1}-\lambda_{2}}{s\left(e^{\lambda_{2} L}-e^{\lambda_{1} L}\right)} \\
\frac{\left(\lambda_{2}-\lambda_{1}\right) e^{\left(\lambda_{1}+\lambda_{2}\right) L}}{s\left(e^{\lambda_{2} L}-e^{\lambda_{1} L}\right)} & \frac{\lambda_{1} e^{\lambda_{1} L}-\lambda_{2} e^{\lambda_{2} L}}{s\left(e^{\lambda_{2} L}-e^{\lambda_{1} L}\right)}
\end{array}\right)
$$

$\lambda_{1}$ and $\lambda_{2}$ are the eigenvalues of $A(s)$, given by, for $i=1,2$ :

$$
\lambda_{i}(s)=\frac{(\alpha-\beta) s+\gamma+(-1)^{i} \sqrt{d(s)}}{2 \alpha \beta}
$$

with $d(s)=(\alpha+\beta)^{2} s^{2}+2[(\alpha-\beta) \gamma+2 \alpha \beta \delta] s+\gamma^{2}$. 
2) Open-loop poles of the system: The poles of this transfer matrix are obtained as the solutions of

$$
s\left(e^{\lambda_{2}(s) L}-e^{\lambda_{1}(s) L}\right)=0 .
$$

There is a pole in zero (the hyperbolic system acts as an integrator) and the other poles verify the following equation:

$$
d(s)=-\frac{4 \alpha^{2} \beta^{2} k^{2} \pi^{2}}{L^{2}}
$$

with $k \in \mathbb{N}^{*}$.

The poles $\left(p_{ \pm k}\right)_{k \in \mathbb{N}^{*}}$ are then given by:

$$
p_{ \pm k}=\frac{2 \alpha \beta}{(\alpha+\beta)^{2}}\left[-\delta-\left(\frac{1}{\beta}-\frac{1}{\alpha}\right) \frac{\gamma}{2} \pm \sqrt{\Delta(k)}\right]
$$

with $\Delta(k)=\delta^{2}-\frac{\gamma^{2}}{\alpha \beta}+\left(\frac{1}{\beta}-\frac{1}{\alpha}\right) \gamma \delta-\frac{k^{2} \pi^{2}(\alpha+\beta)^{2}}{L^{2}}$.

Let $k_{m} \in \mathbb{N}^{*}$ be the greatest integer such that $\Delta\left(k_{m}\right) \geq 0$. Then the poles obtained for $0<k \leq k_{m}$ are negative real, and those obtained for $k>k_{m}$ are complex conjugate, with a constant real part equal to $-\frac{2 \alpha \beta}{(\alpha+\beta)^{2}}\left[\delta+\left(\frac{1}{\beta}-\frac{1}{\alpha}\right) \frac{\gamma}{2}\right]$. The oscillating poles are therefore located on a vertical line in the left half plane. Let us note that when $\gamma=\delta=0$ the poles are located on the imaginary axis.

3) Properties of the transfer matrix: Using the above definitions, we state the following proposition.

Proposition 1: Each element $p_{i j}(s)$ of the transfer matrix $P(s)$ belongs to the Callier-Desoer algebra $\hat{\mathcal{B}}(\sigma)$ iff $\gamma \neq 0$ or $\delta \neq 0$, with $\sigma>-\frac{2 \alpha \beta}{(\alpha+\beta)^{2}}\left[\delta+\left(\frac{1}{\beta}-\frac{1}{\alpha}\right) \frac{\gamma}{2}\right]$.

Indeed, using the closed form expression of the poles of $P(s), p_{i j}(s)$ can be decomposed as an infinite sum [1]:

$$
p_{i j}(s)=\sum_{k=-\infty}^{\infty} \frac{a_{i j}^{(k)}}{s-p_{k}}
$$

Therefore, since $p_{0}=0$ and the other poles have a negative real part, we get:

$$
p_{i j}(s)=\frac{a_{i j}^{(0)}}{s}+\sum_{k \neq 0} \frac{a_{i j}^{(k)}}{s-p_{k}}
$$

Then, $p_{i j}(s)$ is the sum of an integrator and a stable infinite dimensional part belonging to $\hat{\mathcal{A}}_{-}(\sigma)$. Since the stable infinite dimensional part has finitely many poles with real part larger than $\sigma_{1}=-\frac{2 \alpha \beta}{(\alpha+\beta)^{2}}\left[\delta+\left(\frac{1}{\beta}-\frac{1}{\alpha}\right) \frac{\gamma}{2}\right]$, then $p_{i j}(s) \in \hat{\mathcal{B}}(\sigma)$, with $\sigma>\sigma_{1}$ (see theorem 3 in [1]).

Finally, $P(s) \in M(\hat{\mathcal{B}}(\sigma))$, which is the multivariable extension of $\hat{\mathcal{B}}(\sigma)$.

If $\gamma=\delta=0$, the open-loop poles of the system are located on the imaginary axis, thus, following [6] it does not belong to $\hat{\mathcal{B}}(0)$.

\section{Closed-loop transfer matrix}

Let $K(s)$ denote the Laplace transform of the finite dimensional controller $K$, i.e.:

$$
\hat{u}(s)=K(s) \hat{y}(s)+\hat{d}(s)
$$

with $K(s)=\left(\begin{array}{ll}k_{11}(s) & k_{12}(s) \\ k_{21}(s) & k_{22}(s)\end{array}\right)$ and where $\hat{d}=\left(\hat{d}_{1} \hat{d}_{2}\right)^{T}$ is the Laplace transform of the input perturbation.

Then the control input $u$ is given by:

$$
\hat{u}(s)=S_{u} \hat{d}(s)+S_{u} K P_{0} \bar{\xi}_{0}(s, L)
$$

with $S_{u}=(I-K P)^{-1}$ the input sensitivity function and the outputs by:

$$
\hat{y}(s)=P S_{u} \hat{d}(s)+S_{y} P_{0} \bar{\xi}_{0}(s, L)
$$

with $S_{y}=(I-P K)^{-1}$ the output sensitivity function.

If the controller $K(s)$ belongs to $\hat{\mathcal{B}}(\sigma)$, then the feedback interconnection also belongs to $\hat{\mathcal{B}}(\sigma)$ provided $\operatorname{det}(I-K P)$ is bounded away from zero at infinity in $\mathbb{C}_{\sigma^{+}}$. Then, the Nyquist criterion applies, which gives a necessary and sufficient condition of closed-loop input-output stability [6].

1) Stability condition derived from Nyquist criterion:

When the product $P(s) K(s)$ is strictly proper, e.g. as a result of the bandwidth limitation usually imposes strictly proper controllers. In this case, the Nyquist criterion can be used as in the finite dimensional case, to study the closed-loop stability of the controlled system.

However, in some hyperbolic systems where boundary conditions are imposed by physical constraints, one needs to consider non strictly proper diagonal controllers. This case will be considered in section $\mathrm{V}$, due to its practical importance for some systems. Let us note that it is still possible to use Nyquist theorem in this case, but its use is more delicate due to the system behavior when $\omega$ tends to infinity (see [2], [3]).

The Nyquist theorem enables to extend classical results for finite dimensional systems to infinite dimensional systems belonging to the Callier-Desoer algebra. However, it only provides an input-output or external stability result. In the next section, we provide Lyapunov type stability result.

\section{STATE SPACE ANALYSIS}

We now state two results concerning the state behaviour. First the transfer function approach can be generalized by using the distributed transfer function, which relates the inputs to the state $\hat{\xi}(s, x)$. Second, we show that input-output stability of the closed-loop system implies the exponential stability of the state of the system.

\section{A. From input to state}

1) Open-loop distributed transfer matrix: The Laplace transform also enables to derive from eq. (1) the distributed transfer matrix expressing the state of the system $\hat{\xi}(s, x)=$ $(\hat{h}(s, x), \hat{q}(s, x))^{T}$ at each point $x \in[0, L]$ of the system as a function of the boundary and initial conditions:

$$
\begin{aligned}
& \hat{\xi}(s, x)=G(s, x) \hat{u}(s)+G_{0}(s, x) \bar{\xi}_{0}(s, L)+\Gamma(s, x) \bar{\xi}_{0}(s, x) \\
& \text { with } G_{0}(s, x)=G(s, x)\left(\begin{array}{cc}
0 & 1 \\
0 & 0
\end{array}\right)-\Gamma(s, x) \text {, and } \\
& \qquad G(s, x)=\left(\begin{array}{cc}
\frac{\lambda_{2} e^{\lambda_{2} x+\lambda_{1} L}-\lambda_{1} e^{\lambda_{1} x+\lambda_{2} L}}{s\left(e^{\lambda_{2} L}-e^{\lambda_{1} L}\right)} & \frac{\lambda_{1} e^{\lambda_{1} x}-\lambda_{2} e^{\lambda_{2} x}}{s\left(e^{\lambda_{2} L}-e^{\lambda_{1} L}\right)} \\
\frac{e^{\lambda_{1} x+\lambda_{2} L}-e^{\lambda_{2} x+\lambda_{1} L}}{e^{\lambda_{2} L}-e^{\lambda_{1} L}} & \frac{e^{\lambda_{2} x^{x}}-e^{\lambda_{1} x}}{e^{\lambda_{2} L}-e^{\lambda_{1} L}}
\end{array}\right)
\end{aligned}
$$

Using these transfer functions and the boundary controls leads to the closed-loop distributed transfer matrix. 
2) Closed-loop distributed transfer matrix: Using equations (12-14), the distributed closed-loop transfer matrix is written as:

$$
\begin{aligned}
\hat{\xi}(s, x)= & G(s, x) S_{u} \hat{d}(s)+G(s, x) S_{u} N_{0} \bar{\xi}_{0}(s, L) \\
& +\Gamma(s, x)\left[\bar{\xi}_{0}(s, x)-\bar{\xi}_{0}(s, L)\right]
\end{aligned}
$$

with $N_{0}(s)=\left(\begin{array}{ll}0 & 1 \\ 0 & 0\end{array}\right)-K\left(\begin{array}{ll}1 & 0 \\ 0 & 0\end{array}\right)$.

The poles of the closed-loop distributed transfer matrix $G_{K}(s, x)=G(s, x) S_{u}(s) N_{0}(s)$ are identical to the ones of the closed-loop input-output transfer matrix $P(s) S_{u}(s)$, only the zeros change. This is due to the fact that the feedback is applied only at the boundaries. Therefore, the results obtained in the last section for the external stability can be directly generalized to the state, since for any $x$ the transfer $G_{K}(s, x)$ belongs to $H_{\infty}$.

\section{B. Exponential stability}

We now recall a result allowing to strongly relate inputoutput stability and Lyapunov stability for systems possessing a minimal state-space realization. This result finds its roots in the dissipativity framework introduced by Willems in his seminal paper [17].

In the sequel, $\Sigma$ is a causal linear time-invariant system such that for any input $u$ in $\mathcal{L}_{2}\left([0, t], \mathbb{R}^{p}\right)$, its output given by $y=\Sigma(u)$ belongs to $\mathcal{L}_{2}\left([0, t], \mathbb{R}^{m}\right)$ ( $\Sigma$ is thus assumed well-defined). $Z$ is a normed vectorial space equipped with the norm $\|\cdot\|_{Z}$ and corresponds to the the state-space of $\Sigma$. Finally, the state of $\Sigma$ at time $t \in[0, \infty)$ belonging to $Z$ is denoted by $z(t)$ and it is formally related to the input $u$ and the initial condition by the following causal relation: $z(t)=\phi\left(t, 0, z(0), u^{(t)}\right)$.

The following definition corresponds to the uniform reachability and the uniform observability defined by Willems in [16] for causal linear invariant systems.

Definition 3: $\Sigma$ is said to be minimal if its state-space is reachable from $z(0)=0$, i.e., there exists $\alpha_{r}>0$ and $T_{r}>0$ such that for any $z \in Z$ there exists $u_{r} \in \mathcal{L}_{2}\left(\left[0, T_{r}\right], \mathbb{R}^{p}\right)$ such that $z(0)=0, z=z\left(T_{r}\right)=\phi\left(T_{r}, 0,0, u_{r}^{\left(T_{r}\right)}\right)$ and $\int_{0}^{T_{r}}\left\|u_{r}(\tau)\right\|^{2} d \tau \leq \alpha_{r}^{2}\|z\|_{Z}^{2}$ and $\Sigma$ is observable, i.e., there exists $\beta_{o}>0$ and $T_{o}>0$ such that for any $z \in Z$ and $u=0$, we have $\int_{0}^{T_{o}}\|y(\tau)\|^{2} d \tau \geq \beta_{o}^{2}\|z\|_{Z}^{2}$.

Proposition 2: Let $\Sigma$ be a causal linear time invariant system defined from $\mathcal{L}_{2}\left([0, t], \mathbb{R}^{p}\right)$ into $\mathcal{L}_{2}\left([0, t], \mathbb{R}^{m}\right)$. If $\Sigma$ is finite gain stable on $\mathcal{L}_{2}$ and if its state-space realization is minimal then $\Sigma$ is exponentially stable, i.e. there exist $a$ and $b$ positive such that for any $z(0) \in Z$, we have $\|z(t)\|_{Z} \leq a e^{-b t}\|z(0)\|_{Z}$ for any $t \geq 0$.

Proof: The proof is omitted for lack of space.

Actually if the closed-loop system is internally stable then the map between $\left(d_{1}, d_{2}\right)$ to $\left(y_{1}, y_{2}\right)$ is $\mathcal{L}_{2}$ gain stable (since the closed-loop matrix belongs to $H_{\infty}$ ) and thus only the minimality of the state-space realization of the closed-loop operator has to be proved.

In our context, the state-space of the closed-loop system is given by the concatenation of the state-space of the hyperbolic system given by (1) and the one of the controller $K$. We then deduce that $Z=\mathcal{L}_{2}\left([0, L], \mathbb{R}^{2}\right)$ and $z=\xi$ when a constant feedback is considered. When $K$ is a finite dimensional time-invariant linear controller of order $n$, then $Z=\mathcal{L}_{2}\left([0, L], \mathbb{R}^{2}\right) \times \mathbb{R}^{n}$ with $z=\left(\xi, x_{K}\right)$ where $x_{K}$ is the state of $K$. In this last case, $Z$ is equipped with the following norm: $\|z\|_{Z}=\left(\|\xi\|_{\mathcal{L}_{2}\left([0, L], \mathbb{R}^{2}\right)}^{2}+\left\|x_{K}\right\|^{2}\right)^{1 / 2}$.

If we assume that the state-space realization of the controller, $K=\left[\begin{array}{l|l}A & B \\ \hline C & D\end{array}\right]$ is such that $(A, B)$ is controllable and $(A, C)$ is observable, it is straightforward to prove that the state-space realization of $K$ is minimal following definition 3.

Following this preliminary remark, the minimality of closed-loop system is ensured if the hyperbolic system given by (1) is also minimal. That can be easily deduced of results presented in [12] (see also [14]). Actually, the state-space of system (1) is reachable from $\xi_{0}=0$, i.e., there exist two finite constants $T_{r}>0$ and $\alpha_{r}>0$ such that for any $\left.\xi_{1} \in \mathcal{L}_{2}\left([0, L], \mathbb{R}^{2}\right)\right)$ there exists $u \in \mathcal{L}_{2}\left([0, T], \mathbb{R}^{2}\right)$ such that $\xi_{1}\left(T_{r}, \cdot\right)=\Phi\left(T_{r}\right) u^{\left(T_{r}\right)}$ and with $\|u\|_{\mathcal{L}_{2}\left(\left[0, T_{r}\right], \mathbb{R}^{2}\right)} \leq$ $\alpha_{r}\left\|\xi_{1}\left(T_{r}, \cdot\right)\right\|_{\mathcal{L}_{2}\left([0, L], \mathbb{R}^{2}\right)}$. Using the duality between controllability and observability (see e.g. [14]), it is also possible to prove that system (1) is observable, i.e., there exist two finite constants $T_{o}>0$ and $\beta_{o}>0$ such that for any $\xi_{1} \in$ $\left.\mathcal{L}\left([0, L], \mathbb{R}^{2}\right)\right)$, we have $\|y\|_{\mathcal{L}_{2}\left(\left[0, T_{o}\right], \mathbb{R}^{2}\right)} \geq \beta_{o}\left\|\xi_{0}\right\|_{\mathcal{L}_{2}\left([0, L], \mathbb{R}^{2}\right)}$ where $y$ corresponds to the output of system (1) initialized at $\xi(0, \cdot)=\xi_{0}$ and where $u(t)=0$ for $t \in\left[0, T_{o}\right]$.

In conclusion, if the state-space realization of the controller is minimal, we are then able to deduce that the initial condition of the closed-loop system is then forgotten exponentially.

\section{SPECIFIC CASE OF STATIC DIAGONAL BOUNDARY CONTROL}

Proportional diagonal controllers are commonly encountered (gates in the case of open-channels lead to static boundary control), and have been studied in the literature (see e.g. [7]). In this case, the closed-loop system simplifies. We study the poles of the closed-loop system, and derive an analytical necessary and sufficient condition for exponential stabilization with decay rate strictly lower than $\mu$ for proportional diagonal boundary control in the case $\gamma=\delta=0$. In the general case, the closed-loop poles cannot be expressed with closed form solutions, but asymptotic analysis is possible in high frequencies. We then consider a static boundary controller defined by:

$$
K=\left(\begin{array}{cc}
k_{0} & 0 \\
0 & k_{L}
\end{array}\right)
$$

where $k_{0}, k_{L}$ are constant scalars and we want to determine conditions on $\left(k_{0}, k_{L}\right)$ such that the closed-loop system is stable.

\section{A. General case}

Following the remarks done in section III.C, the closedloop system is well-posed, and the Nyquist stability criteria applies, leading to necessary and sufficient condition for stability. 
The resulting closed-loop system is then given by Eq. (13), and $\operatorname{det}(I-P(s) K(s))=e^{\lambda_{1} L}\left(s+k_{0} \lambda_{2}\right)\left(s+k_{L} \lambda_{1}\right)-$ $e^{\lambda_{2} L}\left(s+k_{0} \lambda_{1}\right)\left(s+k_{L} \lambda_{2}\right)$.

The poles of the closed-loop system are therefore solutions of the following equation:

$$
e^{\left(\lambda_{2}(s)-\lambda_{1}(s)\right) L}=\frac{\left(s+k_{0} \lambda_{2}(s)\right)\left(s+k_{L} \lambda_{1}(s)\right)}{\left(s+k_{0} \lambda_{1}(s)\right)\left(s+k_{L} \lambda_{2}(s)\right)}
$$

Unfortunately, there are no closed-form solutions for this equation, but the Nyquist criterion applies, giving a graphical result for closed-loop stability.

It is also possible to derive asymptotic approximation for the poles in high and low frequencies.

In high frequencies, the eigenvalues can be approximated by $\lambda_{1}(s)=-r_{1}-\frac{s}{\alpha}+O\left(\frac{1}{s}\right)$ and $\lambda_{2}(s)=r_{2}+\frac{s}{\beta}+O\left(\frac{1}{s}\right)$, with $r_{1}=\frac{\alpha \delta-\gamma}{\alpha(\alpha+\beta)}$ and $r_{2}=\frac{\beta \delta+\gamma}{\beta(\alpha+\beta)}$.

Therefore, for $|s| \gg \frac{2[(\alpha-\beta) \gamma+2 \alpha \beta \delta]}{(\alpha+\beta)^{2}}$, the high frequency closed-loop poles are approximated by:

$p_{ \pm k} \approx-\frac{\left(r_{1}+r_{2}\right) L}{\tau}+\frac{1}{\tau} \log \left(\frac{\left(\beta+k_{0}\right)\left(\alpha-k_{L}\right)}{\left(\alpha-k_{0}\right)\left(\beta+k_{L}\right)}\right) \pm \frac{2 j k \pi}{\tau}$

In low frequencies, we have similarly $\lambda_{1}(s)=-\frac{\delta}{\gamma} s+o(s)$ and $\lambda_{2}(s)=\frac{\gamma}{\alpha \beta}+\left(\frac{1}{\beta}-\frac{1}{\alpha}+\frac{\delta}{\gamma}\right) s+o(s)$. Therefore, for $|s| \ll \frac{\gamma^{2}}{2[(\alpha-\beta) \gamma+2 \alpha \beta \delta]}$, the low frequency closed-loop poles are approximated by:

$p_{ \pm k} \approx-\frac{1}{\gamma(\alpha-\beta)+2 \delta}+\frac{1}{\tau_{0}} \log \left(\frac{k_{0}\left(\gamma-k_{L} \delta\right)}{k_{L}\left(\gamma-k_{0} \delta\right)}\right) \pm \frac{2 j k \pi}{\tau_{0}}$

with $\tau_{0}=\frac{L}{\beta}-\frac{L}{\alpha}+\frac{2 L \delta}{\gamma}$.

These results extend the poles approximations obtained for one boundary control at $x=L$ [9]. However, it is difficult to derive a stability property from an asymptotic frequency behavior of the poles.

\section{B. Case $\delta=\gamma=0$}

We now consider the special case where $\delta=\gamma=0$, which corresponds to the system considered by several authors (see e.g. [7]). In this case, the transfer matrix no longer belongs to the class $\hat{\mathcal{B}}(0)$ and can only be stabilized by a non strictly proper controller [6]. Therefore, the Nyquist criterion does not apply. We can nevertheless show that it belongs to the class of regular transfer functions and then well-posedness of the closed-loop can be guaranteed (see [15] and references therein).

Moreover, a necessary and sufficient condition for closedloop stability can be derived from the closed-form expression for the poles of the closed-loop system.

In this case, the eigenvalues are given by $\lambda_{1}(s)=-\frac{s}{\alpha}$ and $\lambda_{2}(s)=\frac{s}{\beta}$. Then, equation (18) becomes:

$$
e^{\left(\frac{1}{\alpha}+\frac{1}{\beta}\right) s L}=\frac{\left(\beta+k_{0}\right)\left(\alpha-k_{L}\right)}{\left(\alpha-k_{0}\right)\left(\beta+k_{L}\right)}
$$

The closed-loop poles are then obtained in a closed-form:

$$
p_{ \pm k}=\frac{1}{\tau} \log \left(\frac{\left(\beta+k_{0}\right)\left(\alpha-k_{L}\right)}{\left(\alpha-k_{0}\right)\left(\beta+k_{L}\right)}\right) \pm \frac{2 j k \pi}{\tau}
$$

with $\tau=L\left(\frac{1}{\alpha}+\frac{1}{\beta}\right)$ and where the complex form of the logarithm is used.

Therefore, we have the following necessary and sufficient result of input output stability:

Proposition 3: Let $\mu \geq 0$ be a positive real number. The closed-loop poles verify $\Re\left(p_{k}\right)<-\mu$ if and only if the couple $\left(k_{0}, k_{L}\right)$ verifies the following inequality:

$$
\left|\frac{\left(\beta+k_{0}\right)\left(\alpha-k_{L}\right)}{\left(\alpha-k_{0}\right)\left(\beta+k_{L}\right)}\right|<e^{-\mu \tau}
$$

This condition extends the one obtained by [7], as shown below in section VI.

Let us now examine the implications of (20) for specific values of $\left(k_{0}, k_{L}\right)$. When $k_{0}=0$, i.e. for simple boundary control at $x=L$, and for $\mu=0$, the condition (20) reduces to $\left|\frac{1-k_{L} / \alpha}{1+k_{L} / \beta}\right|<1$, which is verified for any $k_{L}>0$. Therefore, any positive proportional boundary controller at $x=L$ stabilizes the system (1). The minimum is obtained for $k_{L}=\alpha$, which is the gain for optimal damping of oscillating modes (see [9]).

When $k_{L}=0$, i.e. for simple upstream boundary control, and for $\mu=0$, the condition reduces to $\left|\frac{1+k_{0} / \beta}{1-k_{0} / \alpha}\right|<1$, which is true for $-\frac{2 \alpha \beta}{\alpha-\beta}<k_{0}<0$. Therefore, contrarily to the boundary control case at $x=L$, the closed-loop system with boundary control at $x=0$ is not stable for any $k_{0}<0$. The minimum is obtained for $k_{0}=-\beta$, which is again the gain for optimal damping of oscillating modes in the case of boundary control at $x=0$.

\section{APPLICATION TO BOUNDARY CONTROL OF AN OPEN-CHANNEL}

\section{A. Linearized Saint-Venant equations}

We apply the result of the paper to the control of a prismatic canal pool of length $L$ with uniform geometry (not necessarily rectangular) and a given bed slope $S_{b} \geq 0$, represented by the linearized Saint-Venant equations involving small variations of discharge $q(t, x)$ and water depth $h(t, x)$ around constant stationary values $Q_{0}\left(\mathrm{~m}^{3} / \mathrm{s}\right)$ and $H_{0}(\mathrm{~m})$.

These equations can be written as a linear hyperbolic system of partial differential equations (1) with the following parameters: $\alpha=C_{0}+V_{0}, \beta=C_{0}-V_{0}, \gamma=$ $g S_{b}\left(\frac{10}{3}-\frac{4 A_{0}}{3 T_{0} P_{0}} \frac{d P_{0}}{d H}\right)$ and $\delta=\frac{2 g S_{b}}{V_{0}}$, where $V_{0}$ is the average velocity, $C_{0}=\sqrt{g A_{0} / T_{0}}$ is the wave celerity, $A_{0}$ the wetted area, $T_{0}$ the water surface top width, $P_{0}$ the wetted perimeter and $g$ the gravitational acceleration.

Note that the variable $h$ is scaled by a factor $T_{0}$, i.e. Eq. (1) applies in fact to $h^{*}=T_{0} h$, which is denoted $h$ with an abuse of notation.

\section{B. Diagonal proportional control}

1) Case $\gamma=\delta=0$ : We explore the link between our result and the stability condition obtained by [7] in the case of a horizontal frictionless channel. In [7], the control is expressed as :

$$
\begin{aligned}
v(t, 0) & =-2 \alpha_{0} c(t, 0) \\
v(t, L) & =2 \alpha_{L} c(t, L)
\end{aligned}
$$


where $v$ and $c$ are deviations from equilibrium values of velocity $V_{0}$ and celerity $C_{0}$ and $\alpha_{0}, \alpha_{L}$ are positive constants such that $0<\alpha_{0}<1$ and $0<\alpha_{L}<1$.

Expressed in terms of our boundary conditions, since $v=$ $\frac{q}{H_{0}}-\frac{V_{0}}{H_{0}} h$ and $c=\frac{C_{0}}{2 H_{0}} h$ in rectangular geometry, we get:

$$
\begin{aligned}
\alpha_{0} & =-\frac{1}{C_{0}}\left(k_{0}-V_{0}\right) \\
\alpha_{L} & =\frac{1}{C_{0}}\left(k_{L}-V_{0}\right)
\end{aligned}
$$

where $k_{0}$ and $k_{L}$ are the gains of the boundary controls $q(s, 0)=k_{0} h(s, 0)$ and $q(s, L)=k_{L} h(s, L)$.

Using Eqs. (21-22), it is easy to show that condition (20) is equivalent to:

$$
\left(\frac{1-\alpha_{0}}{1+\alpha_{0}}\right)\left(\frac{1-\alpha_{L}}{1+\alpha_{L}}\right)<e^{-\mu \tau}
$$

For $\mu=0$, i.e. only for stabilization, we recover the sufficient condition obtained by [7] based on a Riemann invariants approach. The frequency domain approach provides here a necessary and sufficient condition for stability.

2) General case: The paper is illustrated for a canal pool of length $L=3000 \mathrm{~m}$ with a trapezoidal geometry, (bed width of $7 \mathrm{~m}$, side slope of 1.5), a bed slope $S_{b}=0.0001$ and Manning coefficient of 0.02 . The considered stationary regime corresponds to a discharge $Q_{0}=14 \mathrm{~m}^{3} / \mathrm{s}$ and a water depth $H_{0}=2.12 \mathrm{~m}$. This leads to an hyperbolic system (1) with the following parameters $\alpha=4.63, \beta=3.33, \gamma=$ $2.7 \times 10^{-3}$, and $\delta=3 \times 10^{-3}$.

Figure 1 depicts the time domain simulation of static diagonal boundary controller for various values of $\left(k_{0}, k_{L}\right)$. The initial state corresponds to a discharge deviation of $0.43 \mathrm{~m}^{3} / \mathrm{s}$ from the equilibrium regime, and initial values of $h(0,0)=0.509 \mathrm{~m}$ and $h(0, L)=0.536 \mathrm{~m}$. The hyperbolic system is simulated with a rational model of order 30 .
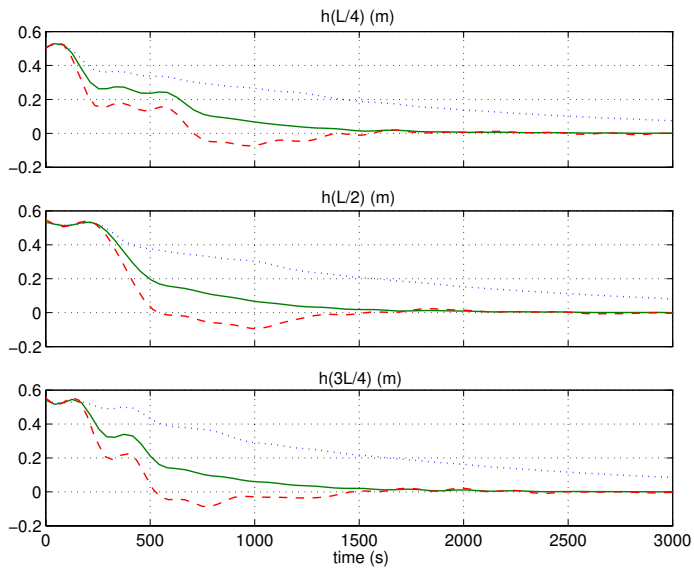

Fig. 1. Water level deviations along time for various values of $\left(k_{0}, k_{L}\right)$ : $(-\beta, \alpha)$ (solid line), $(-\beta / 4, \alpha / 4)$ (dotted line), $(-4 \beta, 4 \alpha)$ (dashed line)

It is clear from the figures that the three controllers stabilize the hyperbolic system, and that the optimal controller leads to the quickest decay of the system.

\section{CONCLUSION}

The paper extends existing results on the stabilization of hyperbolic conservation laws, and proposes a frequency domain approach for the control of such systems. Simulations for boundary control of an open-channel show the effectiveness of the approach. Finally, this paper demonstrates the usefulness of the classical frequency domain approach for analysis and control of distributed parameters systems represented by hyperbolic conservation laws. This preliminary work paves the way towards the study of the stability of the nonlinear Saint-Venant equations for any equilibrium regime.

\section{ACKNOWLEDGMENTS}

The authors thank Joseph Winkin for his comments on the paper and Boumédiène Chentouf for providing useful references.

\section{REFERENCES}

[1] F.M. Callier and J. Winkin. Infinite dimensional system transfer functions, in Analysis and Optimization of Systems: State and Frequency Domain Approaches for Infinite Dimensional Systems, R.F. Curtain, A. Bensoussan and J.L. Lions (eds.), Lecture Notes in Control and Information Sciences, Springer-Verlag, 1992, pp. 72-101.

[2] F.M. Callier and C.A. Desoer. A graphical test for checking the stability of a linear time-invariant feedback system. IEEE Trans. on Automatic Control, 17:773-780, 1972.

[3] F.M. Callier and C.A. Desoer. On symplifying a graphical stability criterion for linear distributed feedback system. IEEE Trans. on Automatic Control, 21:128-129, 1976.

[4] F.M. Callier and C.A. Desoer. An algebra of transfer functions for distributed linear time-invariant systems. IEEE Trans. on Circuits and Systems, CAS-25(9):651-662, 1978.

[5] Ibid. IEEE Trans. on Circuits and Systems, 27(5):320-323, 1980.

[6] R. F. Curtain and H. Zwart. An introduction to infinite dimensional linear systems theory, volume 21 of Text in applied mathematics. Springer Verlag, 1995.

[7] J. de Halleux, C. Prieur, J.-M. Coron, B. d'Andréa Novel, and G. Bastin. Boundary feedback control in networks of open-channels. Automatica, 39:1365-1376, 2003.

[8] X. Litrico and V. Fromion. Frequency modeling of open channel flow. J. Hydraul. Engrg., 130(8):806-815, 2004.

[9] X. Litrico and V. Fromion. Boundary control of linearized SaintVenant equations oscillating modes. Automatica, 42(6):967-972, 2006.

[10] X. Litrico and V. Fromion. $H_{\infty}$ control of an irrigation canal pool with a mixed control politics. IEEE Trans. on Control Systems Technology, 14(1):99-111, 2006.

[11] C. Prieur, J. Winkin, and G. Bastin. Boundary control of nonhomogeneous systems of two conservation laws. In 44th Conf. on Decision and Control, pp. 1899-1904, Sevilla, 2005.

[12] D. L. Russell. Control theory of hyperbolic equations related to certain questions in harmonic analysis and spectral theory. J. Math. Anal. Appl., 40:336-368, 1972.

[13] D. L. Russell. Quadratic performance criteria in boundary control of linear symmetric hyperbolic systems. SIAM Journal on Control, 11(3):475-509, 1973.

[14] D. L. Russell. Controllability and stabilizability theory for linear partial differential equations: recent progress and open questions. SIAM Review, 20(4):639-739, 1978.

[15] G. Weiss. Regular linear systems with feedback. Math. Control Signals Systems, 7:23-57, 1994.

[16] J. C. Willems. The generation of Lyapunov functions for input-output stable systems. SIAM Journal on Control, 9(1):105-134, 1971.

[17] J. C. Willems. Dissipative dynamical systems Part I/Part II. Archive for Rational Mechanics and Analysis, 45:321-341/352-393, 1972. 